\title{
A Mathematical Model of Blood Flow in Merging Veins under a Magnetic Resonance Imaging Influence
}

\author{
W. I. A. Okuyade, T. M. Abbey, M. E. Abbey, and D. M. Abbey
}

\begin{abstract}
Venous blood flow through the superior and inferior vena cavae and via a merger to the right atrium of the heart is investigated. Based on the Newtonian assumption for blood, the model is developed using Boussinesq's approximation for the continuity, momentum, energy, and mass diffusion equations, which are non-linear partial differential equations. The governing equations are non-dimensionalized and solved by the regular perturbation technique. Expressions for the concentration, temperature, and velocity are obtained, analyzed, and presented graphically and quantitatively, and discussed. The results show that an increase in the magnetic field strength and conflux angle produce fluctuations in the flow velocity.
\end{abstract}

Index Terms - merging flow, venous blood, resonance imaging influence.

\section{INTRODUCTION}

Merging flows have found relevance in the engineering and natural worlds. They have applications in the respiratory system, internal machinery, biomechanics, etc.

Blood flow in the human body is pulsatile. The pulsatility arises from the rhythmic beating of the heart, which is due to the wave-like stimulus coming from the sino-arterial node (paceceter), a small part of the right atrium. Being wave-like, the stimulus spreads to the left atrium. The heartbeat or cardiac cycle is in two phases: diastole and systole. During the diastole, the heart muscles are relaxed, and the chambers are filled with blood while in the systole the heart muscles contract, and blood are pumped into the arteries. At the contraction of the arteries, blood presses open the tricuspid and bicuspid valves to fill the ventricles. After this, the valves are closed under the weight of the blood. Furthermore, when the ventricles contract, the deoxygenated blood in the right ventricle is forced into the pulmonary artery that conveys it to the lungs (pulmonary circulation) while the oxygenated blood in the left ventricle is forced into the aorta, which supplies blood to all parts of the body (partly systemic circulation). The force with which blood is pushed into the arteries causes them to expand to accommodate the volume pushed through towards the capillaries. The force which propels the blood gets reduced proportionately with the distance from the heart, implying that it damps out with distance. It is considerably less or low in the venules and veins. The arteries have a mean pressure of approximately

Published on June 22, 2010.

W. I. A. Okuyade, School of Applied Sciences, Federal Polytechnic of Oil and Gas, Bonny, Nigeria.

(e-mail: wiaokuyade ${ }^{\circledR}$ gmail.com)

T. M. Abbey, Applied Mathematics and Theoretical Physics Group, Department of Physics, University of Port Harcourt, Port Harcourt, Nigeria. (e-mail: tamunoimi.abbey ${ }^{\circledR}$ uniport.edu.ng)
$100 \mathrm{mmHg}$ while the veins have approximately $10 \mathrm{mmHg}$ [1]. Upon this, it follows that the force propelling the deoxygenated blood through the veins back to the heart must be a combination of other forces. The expansion of the arteries is the flow pulse, and the force on the wall is the blood pressure, which may be low or high depending on the physiologic situation of the channel and the psychological state of the man.

Additionally, the system of blood vessels starts from the aorta (main artery), which is U-curved and branched to the head, forelimbs, trunk, and hind limbs. These major arterial branches divide severally into arterioles and capillaries. The capillaries have direct contact with the cells and are concerned with materials exchange. From the contact points, the capillaries unite severally to form venules and veins, which carry the deoxygenated blood back to the right atrium of the heart. The major veins concerned with these are the superior and inferior vena cava. The superior vena cava drains the deoxygenated blood from the head and forelimbs and carries it towards the right atrium; the inferior vena cava drains the deoxygenated blood from the trunk and hind limbs and conveys it towards the right atrium. The two veins merged to have a common channel leading to the right atrium. The transport of blood through these two merging veins forms the crux of this work.

A good number of researches have been carried out on the merging flow in both natural and artificial systems in different perspectives. Some worked on the merging flow in hydrologic systems, some on merging flow in blood vessels, and others on merging artificial systems. Importantly, skeletal literature exists on the flow in merging blood vessels, implying that this domain of study has not been fully researched. Amongst the few available reports, [2] studied blood flow through a straight channel with an upstream splitter plate; neglecting the effects of pulsatility, [3] studied a two-dimensional merging blood flow in a basilar artery using geometrical transformation, conformal mapping and numerical approaches; [4] considered the pulsatile blood flow in mesentery arteries with bifurcation and confluence using particle image velocimetry; [5] studied the asymmetry of blood flow and cancer cell adhesion in a micro-channel with symmetric bifurcation and confluence; [6] investigated the effect cell-free layer on the velocity profiles in blood flow in a micro-channel with bifurcation and confluence. Also, there

M. E. Abbey, School of Applied Sciences, Federal Polytechnic of Oil and Gas, Bonny, Nigeria.

(e-mail abbeyminaibim ${ }^{\circledR}$ gmail.com)

D. M. Abbey, School of Applied Sciences, Federal Polytechnic of Oil and Gas, Bonny, Nigeria.

(e-mail: abbeydabebara ${ }^{\circledR}$ gmail.com) 
are some reports on the flow on merging artificial systems. For example, [7] examined a steady two-dimensional asymmetric merging flow of a micro-polar fluid in a rectangular channel using numerical method; [8] examined numerically the simulation of an asymmetric merging flow in a rectangular channel; [9] investigated the influence of magnetic field on the merging flow of Powell-Eyring fluids using an exact solution approach.

It is important to study the flow of blood both in normal or diseased states. Based on the limited existing works of literature on merging flows, we are motivated to study the flow of deoxygenated blood in the merger between the superior and inferior vena cavae when a patient is undergoing Magnetic Resonance Imaging such as ultra-sound, electrocardiogram (ECG), C.T Scan, X-ray, etc., hydrodynamically and bringing into play the effects of some hydrodynamic, physical, and environmental parameters on the flow.

This paper is organized in the following format: section 2 is the methodology, section 3 is the method of solution, section 4 has the results and discussion, and section 5 gives the conclusion.

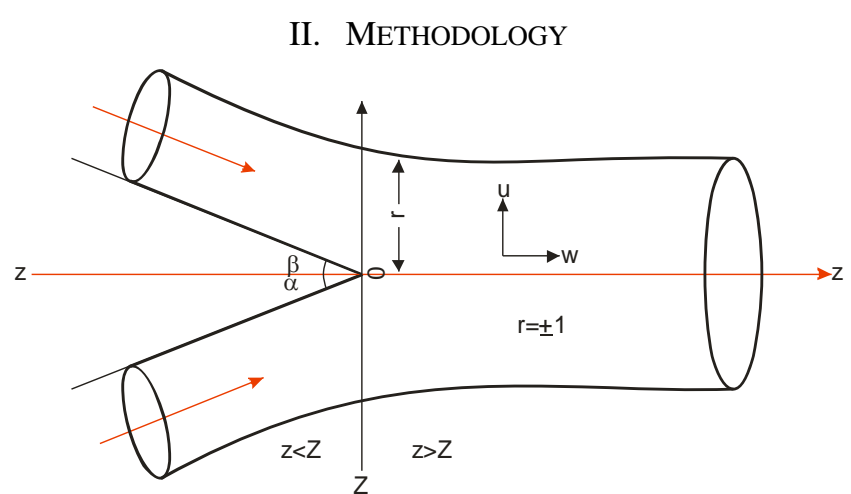

Fig. 1. A schematic of the symmetric merging veins.

We consider the merging flow of deoxygenated blood in the superior and inferior vena cava (veins). The problem is formulated under the assumptions that the pulsatility of the flow is damped out and, therefore neglected; there exist temperature and concentration gradients between the fluid ambient temperature and concentration and the those of the environment; there is a significant interaction with the environment such that the heat exchange effects are noticeable; with the decay of the rhythmic pumping force from the heart, and the presence of the force arising from the temperature and concentration differentials the flow is free convective; the channel is cylindrical and porous; with the significant reduction on the propelling force from the heart, the distensibility of the channels is played down; blood is viscous, incompressible, Newtonian and magnetically susceptible; exposure of the patient to magnetic resonance imaging makes the effect of magnetic field on the problem significant; the flow Reynolds number is greater than unity and as such the inertia force is non-zero; the patient is under the influence of clinical medication, chemical reactions are ignited in the system. Additionally, we assume the velocity is symmetric about the $\theta$-axis such that its variation is zero and the problem becomes two-dimensional. Additionally, since the venous blood from the different vena cavae are under the same conditions, and the channels symmetrical, then only a set of governing equations satisfies the problem. Therefore, if $\left(u^{\prime}, 0, w^{\prime}\right)$ are velocity components in the $\left(r^{\prime}, 0, z^{\prime}\right)$ orthogonal coordinates; $T^{\prime}$ and $C^{\prime}$ are the fluid temperature and concentration, $T_{w}$ and $C_{w}$ are the constant temperature and concentration at the wall, $T_{\infty}$ and $C_{\infty}$ are the fluid equilibrium temperature and concentration, then the governing equations of continuity, momentum, energy, and diffusion, using the Boussinesq approximations are:

$$
\begin{aligned}
& \frac{1}{r} \frac{\partial}{\partial r}\left(r^{\prime} u^{\prime}\right)+\frac{\partial w^{\prime}}{\partial z^{\prime}}=0 \\
& \rho\left(u^{\prime} \frac{\partial u^{\prime}}{\partial r^{\prime}}+w^{\prime} \frac{\partial u^{\prime}}{\partial z^{\prime}}\right)=-\frac{\partial p^{\prime}}{\partial r^{\prime}}+\mu\left(\frac{\partial^{2} u^{\prime}}{\partial r^{\prime 2}}+\frac{1}{r^{\prime}} \frac{\partial u^{\prime}}{\partial r^{\prime}}-\frac{u^{\prime}}{r^{\prime 2}}+\frac{\partial^{2} u^{\prime}}{\partial z^{\prime 2}}\right)
\end{aligned}
$$

$$
\begin{aligned}
& \rho\left(u^{\prime} \frac{\partial w^{\prime}}{\partial r^{\prime}}+w^{\prime} \frac{\partial w^{\prime}}{\partial z^{\prime}}\right)=-\frac{\partial p^{\prime}}{\partial z^{\prime}}+\mu\left(\frac{\partial^{2} w^{\prime}}{\partial r^{\prime 2}}+\frac{1}{r^{\prime}} \frac{\partial w^{\prime}}{\partial r^{\prime}}+\frac{\partial^{2} w^{\prime}}{\partial z^{\prime 2}}\right)- \\
& \rho g \beta_{1}\left(T^{\prime}-T_{\infty}\right)-\rho g \beta_{2}\left(C^{\prime}-C_{\infty}\right)-\left(\frac{\mu}{\kappa}+\frac{\sigma_{e} B_{o}^{2}}{\rho \mu \mu_{m}}\right) w^{\prime}
\end{aligned}
$$

$\rho C_{p}\left(u^{\prime} \frac{\partial T^{\prime}}{\partial r^{\prime}}+w^{\prime} \frac{\partial T^{\prime}}{\partial z^{\prime}}\right)=-k\left(\frac{\partial^{2} T^{\prime}}{\partial r^{\prime 2}}+\frac{1}{r^{\prime}} \frac{\partial T^{\prime}}{\partial r^{\prime}}+\frac{\partial^{2} T^{\prime}}{\partial z^{\prime 2}}\right)+Q\left(T^{\prime}-T_{\infty}\right)$

$$
u^{\prime} \frac{\partial C^{\prime}}{\partial r^{\prime}}+w^{\prime} \frac{\partial C^{\prime}}{\partial z^{\prime}}=D\left(\frac{\partial^{2} C^{\prime}}{\partial r^{\prime 2}}+\frac{1}{r^{\prime}} \frac{\partial C^{\prime}}{\partial r^{\prime}}+\frac{\partial^{2} C^{\prime}}{\partial z^{\prime 2}}\right)+k_{r}^{2}\left(C^{\prime}-C_{\infty}\right)
$$

From the schematic, Fig. 1 it can be seen that the channel is symmetrical and split into two regions: the upstream $z<Z$ and downstream $z>Z$, where $Z$ is the nodal or merging point taking as the origin such that the boundaries become $r^{\prime}=\alpha z^{\prime}$ for the upstream and $r^{\prime}= \pm 1$ the downstream. By geometrical transition between the upstream and downstream regions of the channel, the problem of wall curvature exists. To fix up this, a simple transition wherein the diameter of the upstream region is made equal to half that of the downstream region such that the variation of the merging angle is used directly [10]. Furthermore, the diameter of the downstream is assumed to be far less than the length such that $\frac{d}{l}=\psi<<1$ where $\psi$ is the aspect ratio $[10,11]$, thus suggesting that the flow is laminar and Poiseuille. Based on the above, the boundary conditions become:

in the upstream, $z<Z$ :

$$
\begin{aligned}
& u^{\prime}=0, w^{\prime}=0, T^{\prime}=0, C^{\prime}=0 \text { at } r^{\prime}=0 \\
& u^{\prime}=0, w^{\prime}=0, T^{\prime}=\xi_{1} T_{w}, C^{\prime}=\xi_{2} C_{w}, \xi_{1}=\xi_{2}<1 \quad \text { at } \quad r=\alpha z^{\prime}
\end{aligned}
$$

and in the downstream, $z>Z$ :

$u^{\prime}=1, w^{\prime}=1, T^{\prime}=T_{\infty}, C^{\prime}=C_{\infty}$ at $r^{\prime}=0$

$u^{\prime}=0, w^{\prime}=0, T^{\prime}=T_{w}, C^{\prime}=C_{w}$ at $r^{\prime}=1$ 
Introducing the non-dimensionalized quantities into equations (1)-(9), gives:

$\frac{1}{r} \frac{\partial(r u)}{\partial r}+\frac{\psi \partial w}{\partial z}=0$

$\frac{\partial^{2} u}{\partial r^{2}}+\frac{1}{r} \frac{\partial u}{\partial r}-\frac{u}{r^{2}}+\frac{\psi^{2} \partial^{2} w}{\partial z^{2}}=\frac{\partial p}{\partial r}+u \frac{\partial u}{\partial r}+\psi w \frac{\partial u}{\partial z}$

$\frac{\partial w}{\partial r}+\frac{1}{r} \frac{\partial w}{\partial r}+\frac{\psi^{2} \partial^{2} w}{\partial z^{2}}-\left(M^{2}+\chi^{2}\right) w=\psi \frac{\partial p}{\partial z}+u \frac{\partial w}{\partial r}+\psi w \frac{\partial w}{\partial z}+G r \Theta+G c \Phi$

$\frac{\partial^{2} \Theta}{\partial r^{2}}+\frac{1}{r} \frac{\partial \Theta}{\partial r}+\frac{\psi^{2} \partial^{2} \Theta}{\partial z^{2}}+N^{2} \Theta=P e_{h}\left(u \frac{\partial \Theta}{\partial r}+\psi w \frac{\partial \Theta}{\partial z}\right)$

$\frac{\partial^{2} \Phi}{\partial r^{2}}+\frac{1}{r} \frac{\partial \Phi}{\partial r}+\frac{\psi^{2} \partial^{2} \Phi}{\partial z^{2}}+\delta^{2} \Phi=P e_{m}\left(u \frac{\partial \Phi}{\partial r}+\psi w \frac{\partial \Phi}{\partial z}\right)$

with the boundary conditions:

$u=0, w=0, \Theta=0, \Phi=0$ at $r=0$

$\mathrm{u}=0, \mathrm{w}=0, \Theta=\xi_{1} \Theta_{w}, \Phi=\xi_{2} \Phi_{w}, \quad \xi_{2}, \xi_{2}<1$ at $r=\psi \alpha<$

for the upstream:

$u=1, \quad w=1, \Theta=1, \Phi=1$ at $\mathrm{r}=0$

$\mathrm{u}=0, \mathrm{w}=0, \Theta=\Theta_{w}, \Phi=\Phi_{w}$ at $\mathrm{r}=1$

and for the downstream

where

$r=\frac{r^{\prime}}{R_{o}}, z=\frac{\psi z^{\prime}}{l}, \Theta=\frac{T^{\prime}-T_{\infty}}{T_{w}-T_{\infty}}, \Phi=\frac{C^{\prime}-C_{\infty}}{C_{w}-C_{\infty}}, w=\frac{w^{\prime} R_{o}}{l}$,

$p=\frac{\left(p^{\prime}-p_{\infty}\right) r_{o}^{3}}{\rho l v}, \operatorname{Re}=v l, M^{2}=\frac{\sigma_{e} B_{o}^{2}}{\rho \mu \mu_{m}}$

$N^{2}=\frac{Q}{k_{o}}, \chi^{2}=\frac{R_{o}}{\kappa}, \delta_{1}^{2}=\frac{k_{r}^{2}}{D}$,

$S c=\frac{v}{D}, \operatorname{Pr}=\frac{\mu C p}{k_{o}}, G r=\frac{g \beta_{1}\left(T_{w}-T_{\infty}\right)}{v^{2}}$,

$G c=\frac{g \beta_{2}\left(C_{w}-C_{\infty}\right)}{v^{2}}, P e_{m}=\operatorname{Re} S c P e_{h}=\operatorname{Re} \operatorname{Pr}$

Equations (11)-(14) are highly coupled and non-linear. Attempting to make them tractable, we assume that with $\psi<<1$, its square will be small negligible enough and that the flow is fully developed such that $u=u(r)=0$. Upon these, our working equations reduce to:

$\frac{\partial w}{\partial z}=0$ $0=\frac{\partial p}{\partial r}$

$\frac{\partial w}{\partial r}+\frac{1}{r} \frac{\partial w}{\partial r}-\left(M^{2}+\chi^{2}\right) w=\psi \frac{\partial p}{\partial z}+\psi w \frac{\partial w}{\partial z} \quad+G r \Theta+G c \Phi$

$\frac{\partial^{2} \Theta}{\partial r^{2}}+\frac{1}{r} \frac{\partial \Theta}{\partial r}+N^{2} \Theta=P e_{h} \psi w \frac{\partial \Theta}{\partial z}$

$\frac{\partial^{2} \Phi}{\partial r^{2}}+\frac{1}{r} \frac{\partial \Phi}{\partial r}-\delta_{1}^{2} \Phi=P e_{m} \psi w \frac{\partial \Phi}{\partial z}$

where $M^{2}=M^{2}+\chi^{2}-\gamma^{2}$ with the boundary conditions as in equations (15)-(18) still holding.

\section{METHOD OF SOLUTION}

A check on equations (21)-(23) shows that they are still very coupled and non-linear. To further linearize and make them tractable, we seek for perturbation series solutions of the form:

$g(r, z)=g^{(o)}(r, z)+\psi g^{(1)}(r, z)+\psi^{2} g^{(2)}(r, z)+\ldots$

where $\psi<<1$ is the perturbation parameter. Furthermore, we choose $g^{(o)}(r, z)=g^{(o o)}(r)-\gamma z$ and $g^{(1)}(r, z)=g^{(10)}(r)-\gamma z$, $p(z)=K z-\frac{K_{1} z^{2}}{\psi}$, where $K z$ is the pressure in the upstream $\frac{K_{1} z^{2}}{\psi}$ is that in the downstream, $\gamma, K$ and $K_{1}$ are constants see [6]. These, in conjunction with equation (24) split equations (21)-(23) and (15)-(18) into:

Zeroth order:

$\frac{\partial w^{(o o)}}{\partial z}=0$

$\frac{\partial w^{(o o)}}{\partial r}+\frac{1}{r} \frac{\partial w^{(o o)}}{\partial r}-M_{1}^{2} w^{(o o)}=K+G r \Theta^{(o o)}+G c \Phi^{(o o)}$

$\frac{\partial^{2} \Theta^{(o o)}}{\partial r^{2}}+\frac{1}{r} \frac{\partial \Theta^{(o o)}}{\partial r}+N^{2} \Theta^{(o o)}=0$

$\frac{\partial^{2} \Phi^{(0 o)}}{\partial r^{2}}+\frac{1}{r} \frac{\partial \Phi^{(o o)}}{\partial r}+\delta^{2} \Phi^{(o o)}=0$

with the boundary conditions:

$w^{(o o)}=0, \Theta^{(o o)}=0, \Phi^{(o o)}=0$ at $r=0$

$w^{(o o)}=0, \quad \Theta^{(o o)}=\xi_{1} \Theta_{w}, \Phi^{(o o)}=\xi_{2} \Phi_{w}, \quad \xi_{2}, \quad \xi_{2}<1 \quad$ at $r=\psi O z$

First order: 
$\frac{\partial w^{(10)}}{\partial z}=0$

$\frac{\partial w^{(10)}}{\partial r}+\frac{1}{r} \frac{\partial w^{(10)}}{\partial r}-M_{1}^{2} w^{(10)}=-K_{1} z+w^{(o o)} \frac{\partial w^{(o o)}}{\partial z}+G r \Theta^{(10)}+G c \Phi^{(10)}$

$\frac{\partial^{2} \Theta^{(10)}}{\partial r^{2}}+\frac{1}{r} \frac{\partial \Theta^{(10)}}{\partial r}+N^{2} \Theta^{(10)}=-P e_{h} \gamma \psi w^{(o o)}$

$\frac{\partial^{2} \Phi^{(10)}}{\partial r^{2}}+\frac{1}{r} \frac{\partial \Phi^{(10)}}{\partial r}+\delta^{2} \Phi^{(10)}=-P e_{m} \gamma \psi w^{(o)}$

where $M_{1}^{2}=M^{2}+\chi^{2}-\gamma^{2}$ with the boundary conditions:

$w^{(1)}=1, \Theta^{(1)}=1, \Phi^{(1)}=1$ at $r=0$

$w^{(1)}=0, \Theta^{(1)}=\Theta_{w}, \Phi^{(1)}=\Phi_{w}$ at $\mathrm{r}=1$

An examination of equations (25)-(30) and (31)-(36) depicts that they are cylindrical equations, and their solutions are of the Bessel form. At this point $r=0$ the solutions are empty. Analytically, at this point, the Bessel function of the second kind is infinite. The constant associated with the Bessel function of the first kind is set to be unity to save the solutions from being empty; see [12]. Solving them, using the Mathematica Computational software, the solutions are still empty. It becomes necessary to shift the boundary condition $r=0$ infinitesimally to $r=0.001$, a point not exactly zero but highly approximately zero to obtain non-empty solutions.

\section{RESULTS AND DISCUSSION}

The problem of merging steady flow of venous blood in the vena cavae towards the heart is considered. The effects of M2, Heat exchange parameters, merging angle on the flow are investigated. The computation was done using Mathematica 11.2 Computational software. For constant values of $\quad \gamma=2.0 ; \quad K=0.5 ; \quad K_{1}=0.5 ; \quad \xi_{1}=0.1$; $P_{e h}=P_{e m}=0.2 ; \quad z=1 ; \quad G r=G r=0.2 ; \quad \xi_{2}=0.1 ;$ $\Theta_{w}=\Phi_{w}=3.0 ; \psi=0.01$ increasingly varied values of $\alpha=10,15,20,25,30 ; \quad N^{2}=0.2,0.4,0.6,0.8,1.0,1.2$; $\chi^{2}=0.2,0.4,0.6,0.8,1.0,1.2$ we obtained the results shown in Fig. 1-6 and Table I-III.

Fig. 2 shows that the concentration of the blood fluid decreases as the merging angle increases. Merging angle is a function of area, which in turn is a function of concentration. The larger the area the lower the concentration. Thus, this accounts for what is seen in Fig. 2.

Fig 3 shows that an increase in merging angle produces sinusoidal profiles in the concentration structure for the real case wherein in the crest the profiles decrease as the merging angle increases while in the trough it increases as the merging angle increases.

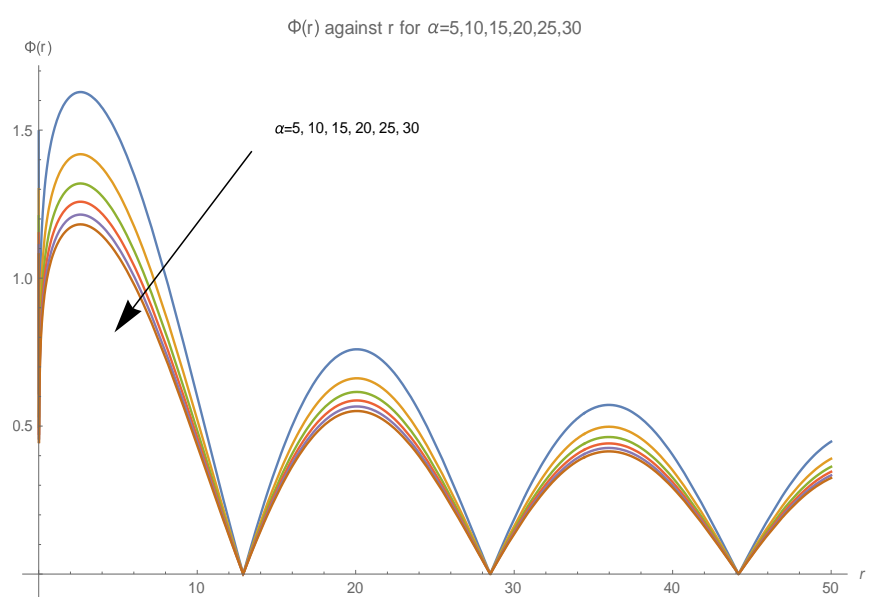

Fig. 2. Concentration-Merging angle $(\alpha)$ profiles for the absolute case.

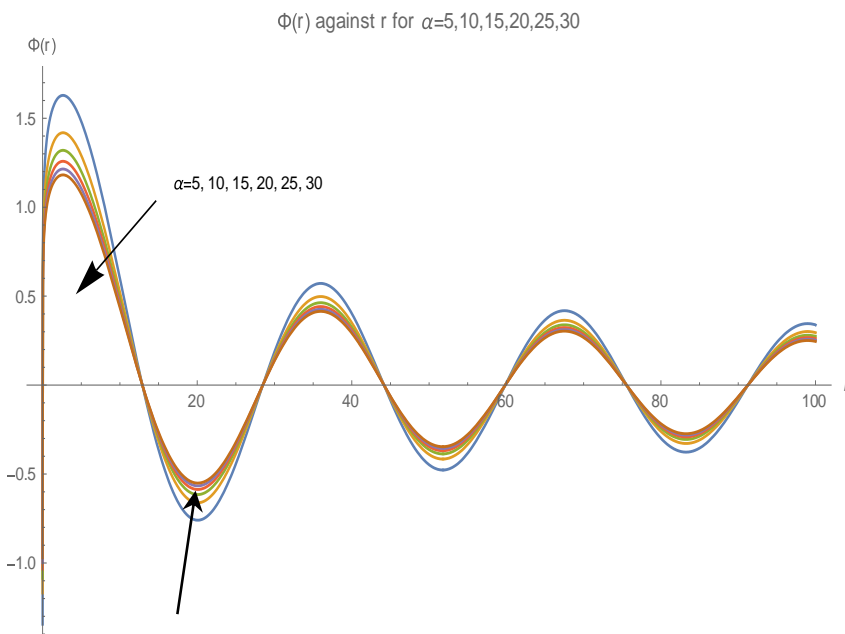

Fig 3. Concentration-Merging angle $(\alpha)$ - Concentration profiles for a real case.

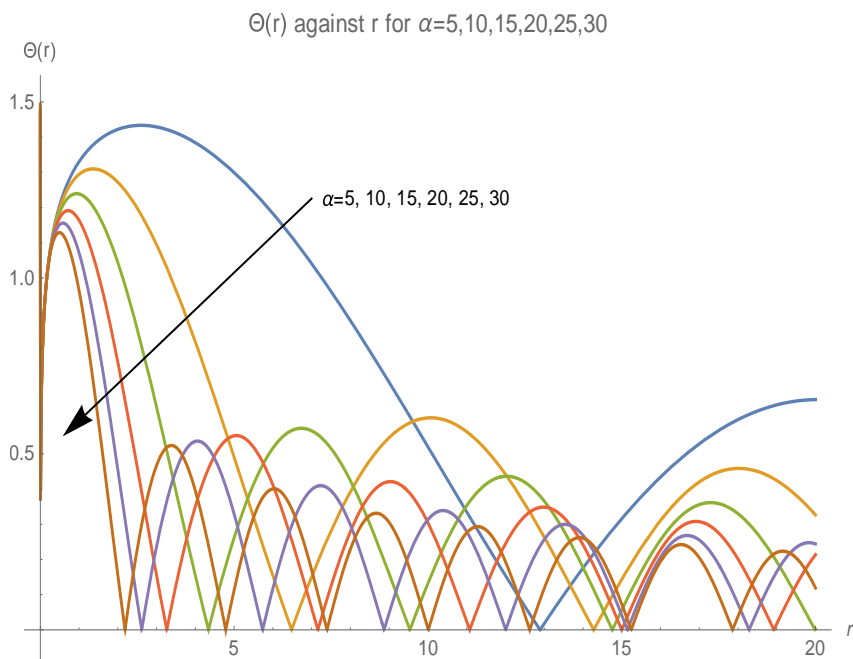

Fig. 4. Temperature-Merging angle ( $\alpha$ ) profiles for the absolute case.

Fig. 4 shows that an increase in the merging decreases the blood temperature, and the profiles are periodic for the absolute case. 


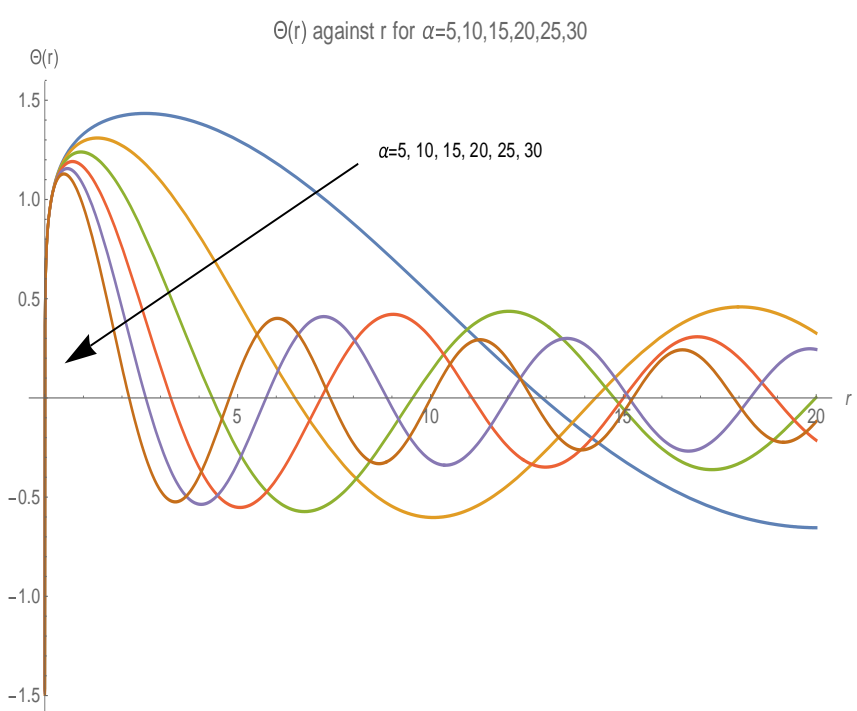

Fig. 5. Temperature-Merging angle ( $\alpha$ ) profiles for the real case.

Fig.5 shows that the flow temperature decreases with the increase in merging angle for the real case and the profiles sinusoidal.

From Fig. 4 and Fig. 5, the oscillatory behaviour seen may lead to loss of energy for the flow.

Additionally, Table I shows that the velocity decreases with the increase in the merging angle. The meeting of the blood from the different channels at the merging point produces some whirling and rotatory motions, which weakens the velocity. This may account for the decrease in the flow velocity.

More so, the oscillatory behavior seen in the concentration and temperature structures, and which reduces the flow strength of these factors also affects the velocity.

Fig. 6 shows that the blood temperature decreases with the increase in the heat exchange parameter $\mathrm{N}^{2}$ increases, and the structures are periodic. There is heat interaction between man and his environment. The environmental heat level depends on the radiation from the sun. Usually, in the interaction, heat is absorbed into the human body and blood stream. Therefore, the reduction in the temperature may be due to other situations in the body.

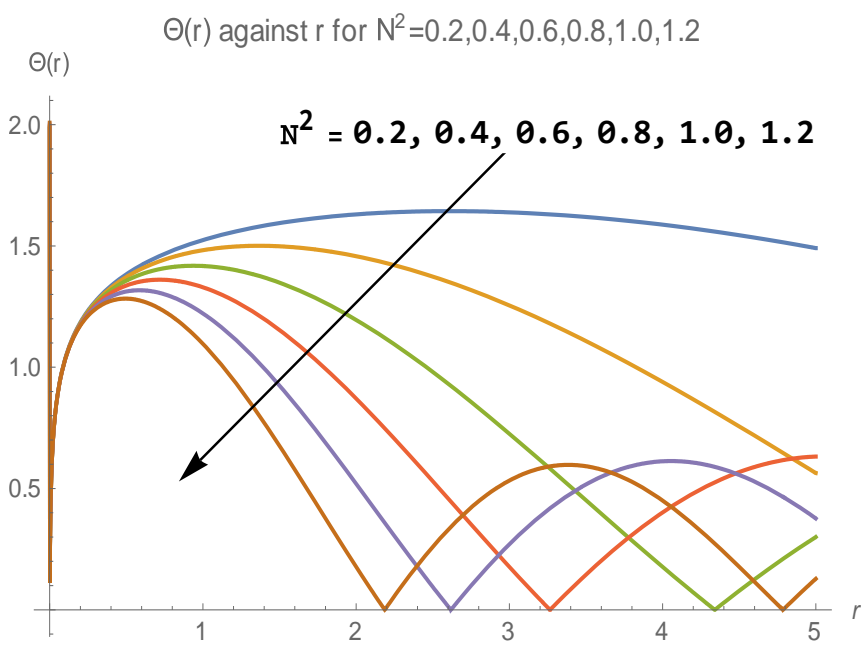

Fig. 6. Temperature-Heat ex-change $\left(\mathrm{N}^{2}\right)$ profiles for the absolute case

Table II shows that an increase in the Heat exchange parameter does not affect the flow velocity. Cuing from the reducing effect of Heat exchange parameter on the temperature; as giving in Fig. 6, velocity as a function of temperature ought to drop as the temperature drops in the presence of increasing merging angle. Therefore, the decrease in the flow velocity in the presence of positively varying Heat exchange parameters may be due to other factors.

Table III shows that increase in the porosity parameter produces fluctuation in the flow velocity structure. At some points, it decreases and at some other points, it rises as the porosity parameter increases. However, the velocity increases as the radius increases.

TABLE I: VELOCITY-MERGING ANGLE (A) RELATION

\begin{tabular}{ccccccc}
\hline \hline & $\mathrm{W}(\alpha=5)$ & $\mathrm{W}(\alpha=10)$ & $\mathrm{W}(\alpha=15)$ & $\mathrm{W}(\alpha=20)$ & $\mathrm{W}(\alpha=25)$ & $\mathrm{W}(\alpha=30)$ \\
\hline $\mathrm{W}$ & $\infty$ & $\infty$ & $\infty$ & $\infty$ & $\infty$ & $\infty$ \\
0.5 & 23.1230 & 12.0696 & 5.5859 & 2.4092 & 0.9994 & 0.4049 \\
1.0 & 2392.4971 & 1248.3922 & 577.7651 & 249.1867 & 103.3698 & 41.8790 \\
1.5 & 288599.78 & 150589.77 & 69694.05 & 30058.64 & 12469.19 & 5051.739 \\
2.0 & $3.7011 \times 10^{7}$ & $1.9312 \times 10^{7}$ & $8.9379 \times 10^{6}$ & $3.8549 \times 10^{6}$ & $1.5991 \times 10^{6}$ & 647859.6 \\
2.5 & $4.9066 \times 10^{9}$ & $2.5603 \times 10^{9}$ & $1.1849 \times 10^{9}$ & $5.1104 \times 10^{8}$ & $2.1200 \times 10^{8}$ & $8.5887 \mathrm{x}^{7}$ \\
3.0 & $6.6419 \times 10^{11}$ & $3.4658 \times 10^{11}$ & $1.6039 \times 10^{11}$ & $6.9177 \times 10^{10}$ & $2.8697 \times 10^{10}$ & $1.1626 \times 10^{10}$ \\
\hline \hline
\end{tabular}

TABLE II: VELOCITY-HEAT EXCHANGE $\left(\mathrm{N}^{2}\right)$ RELATION

\begin{tabular}{ccccccc}
\hline \hline & $\mathrm{W}(\mathrm{N}=\sqrt{0.2})$ & $\mathrm{W}(\mathrm{N}=\sqrt{0.4})$ & $\mathrm{W}(\mathrm{N}=\sqrt{0.6})$ & $\mathrm{W}(\mathrm{N}=\sqrt{0.8})$ & $\mathrm{W}(\mathrm{N}=\sqrt{1.0})$ & $\mathrm{W}(\mathrm{N}=\sqrt{1.2})$ \\
\hline $\mathrm{W}$ & Indeterminate & Indeterminate & Indeterminate & Indeterminate & Indeterminate & Indeterminate \\
0.5 & 12.2665 & 12.2665 & 12.2665 & 12.2665 & 12.2665 & 12.2665 \\
1.0 & 131953. & 131953. & 131953. & 131953. & 131953. & 131953. \\
1.5 & $1.87526 \times 10^{9}$ & $1.87526 \times 10^{9}$ & $1.87526 \times 10^{9}$ & $1.87526 \times 10^{9}$ & $1.87526 \times 10^{9}$ & $1.87526 \times 10^{9}$ \\
2.0 & $3.0526 \times 10^{13}$ & $3.0526 \times 10^{13}$ & $3.0526 \times 10^{13}$ & $3.0526 \times 10^{13}$ & $3.0526 \times 10^{13}$ & $3.0526 \times 10^{13}$ \\
2.5 & $5.33377 \times 10^{17}$ & $5.33377 \times 10^{17}$ & $5.33377 \times 10^{17}$ & $5.33377 \times 10^{17}$ & $5.33377 \times 10^{17}$ & $5.33377 \times 10^{17}$ \\
3.0 & $9.70213 \times 10^{21}$ & $9.70213 \times 10^{21}$ & $9.70213 \times 10^{21}$ & $9.70213 \times 10^{21}$ & $9.70213 \times 10^{21}$ & $9.70213 \times 10^{21}$ \\
\hline \hline
\end{tabular}

TABLE III: VELOCITY-MAGNETIC FIELD (M2) RELATION

\begin{tabular}{ccccccc}
\hline \hline & $\mathrm{W}(\mathrm{M}=\sqrt{0.2})$ & $\mathrm{W}(\mathrm{M}=\sqrt{0.4})$ & $\mathrm{W}(\mathrm{M}=\sqrt{0.6})$ & $\mathrm{W}(\mathrm{M}=\sqrt{0.8})$ & $\mathrm{W}(\mathrm{M}=\sqrt{1.0})$ & $\mathrm{W}(\mathrm{M}=\sqrt{1.2})$ \\
\hline $\mathrm{W}$ & $\infty$ & $\infty$ & $\infty$ & $\infty$ & $\infty$ & $\infty$ \\
0.5 & 0.2092 & 0.1249 & 0.0873 & 0.0661 & 0.0531 & 0.0453 \\
1.0 & 0.3895 & 0.2611 & 0.2211 & 0.2125 & 0.2209 & 0.2417 \\
1.5 & 0.5909 & 0.4397 & 0.4236 & 0.4652 & 0.5534 & 0.6955 \\
2.0 & 0.7991 & 0.6570 & 0.7167 & 0.9025 & 1.2440 & 1.8301 \\
2.5 & 1.0166 & 0.9293 & 1.1583 & 1.6941 & 2.7474 & 4.8070 \\
3.0 & 1.2484 & 1.2800 & 1.8425 & 3.1675 & 6.1186 & 12.8804 \\
\hline \hline
\end{tabular}




\section{CONCLUSION}

A conclusion section is not required. Although a conclusion may review the main points of the paper, do not replicate the abstract as the conclusion. A conclusion might elaborate on the importance of the work or suggest applications and extensions.

\section{NOMENCLATURE}

$C^{\prime} \quad$ Concentration (quantity of material being transported)
$D \quad$ Diffusion coefficient
$g \quad$ Gravitational field vector
Gc Grashof number due to concentration difference
Gr Grashof number due to temperature difference
$K \quad$ Constant body temperature
$K_{1} \quad$ a constant
$p^{\prime} \quad$ Fluid pressure
$p \quad$ Dimensionless pressure
Pr Prandtl number
$Q \quad$ Heat absorption/generation coefficient
Re Reynolds number,
$r_{o} \quad$ Characteristic radius of the capillary
Sc Schmidt number
$T^{\prime} \quad$ Fluid temperature

$\left(u, w^{\prime}\right)$ Velocity components of the fluid in the mutually orthogonal axes

$\begin{array}{ll}(u, w) & \text { Non-dimensionalized velocity components } \\ \left(r^{\prime}, z^{\prime}\right) & \text { Mutually orthogonal axes } \\ (r, z) & \text { Dimensionless orthogonal axes } \\ \alpha, \beta & \text { Bifurcation angles } \\ \rho^{\prime} & \text { Density of the fluid } \\ \rho & \text { Dimensionless density of the fluid } \\ \mu & \text { Viscosity of the fluid } \\ \sigma & \text { Stefan-Botzmann constant, } \\ \sigma_{e} & \text { Electrical conductivity of the fluid } \\ \gamma & \text { Mean radiation coefficient (or depth of penetration }\end{array}$

of the radiant ray)

$\mu_{m} \quad$ Magnetic permeability of the fluid

$\kappa \quad$ Permeability of the porous medium

$v \quad$ Kinematic viscosity of the fluid

$\chi^{2} \quad$ Local Darcy number

$\delta_{1}^{2} \quad$ Rate of chemical reaction

$\Theta \quad$ Dimensionless temperature

$\psi \quad$ Aspect ratio

$\Phi \quad$ Dimensionless concentration

$B_{c} \quad$ Volumetric expansion coefficient due to concentration

$B_{o}^{2} \quad$ Applied uniform magnetic field strength

$B_{t} \quad$ Volumetric expansion coefficient due to temperature

$C_{p} \quad$ Specific heat capacity at constant pressure
$C_{w} \quad$ Constant wall temperature maintained

$C_{\infty} \quad$ Concentration at equilibrium

$k \quad$ Thermal conductivity of the medium

$k_{r}^{2} \quad$ Rate of chemical reaction

$\ell_{c} \quad$ Scale length

$\mathrm{M}^{2} \quad$ Hartmann's number

$\mathrm{N}^{2} \quad$ Heat exchange parameter

$\mathrm{Pe}_{\mathrm{h}} \quad$ Peclet number due to heat transfer

$\mathrm{Pe}_{\mathrm{m}} \quad$ Peclet number due to mass transfer

$p_{\infty} \quad$ Ambient/equilibrium pressure

$T_{w} \quad$ Constant wall concentration at which the channel is maintained

$T_{\infty} \quad$ Temperature at equilibrium

$U_{o} \quad$ Characteristic velocity of the flow

\section{ACKNOWLEDGMENT}

The equations used in this work were culled from Okuyade and Abbey (2017) under the Creative Common Attribution License.

\section{REFERENCES}

[1] D.M. Wooton, and D.N. Ku, (1999). Fluid mechanics of vascular systems, diseases and thrombosis. Annual Review Biomedical Engineering, 299-329.

[2] H. Badre, S.C.R. Dennis, and F.T. Smith. (1985). Numerical and asymptotic solutions for merging flow through a channel with an upstream splitter plate. Journal of Fluid Mechanics, 156, 63-81.

[3] J.K. Krijger, and B. Hillen. (1990). Steady two-dimensional merging flow from two channels into a single channel. Applied Scientific Research, 47(3), 233-246.

[4] Ayushi Nakano, Yusuhiko Sugii, Mobomu Minamiyano (2005). Velocity profiles of pulsatile blood flow in arteries with bifurcation and confluence in rat mesentery measured by particle image velocimetry. Bifurcation and Confluence, JSME International Journal Series C, 48 (4), 444-452. Doi 10.1299/jsmec 48.444.

[5] T. Ishikawa, H. Fujiwara, N. Matsuki, T. Yoshimoto, Y. Imai, H. Ueno, and T. Yamaguchi (2011). Asymmetry of blood flow and cancer cell adhesion in a micro-channel with symmetric bifurcation and confluence, Biomedical Micro-devices, 13, 159-167.

[6] D. Pinho, D. Bento, and R. Lima. (2014) studied the effect of cell-free layer on the velocity profiles in blood flow in a micro-channel with bifurcation and confluence, Material Science, Corpus ID: 137708448.

[7] A.A. Siddiqui. (2013). Numerical Simulation of an asymmetric merging flow in a rectangular channel, arXiv: 1308.0805 (Physics).

[8] Siddiqui, A.A. (2016), Numerical simulation of asymmetric merging flow in a rectangular channel. World Journal of Mechanics. 6, 118-130. http://dx.doi.org/10.4236/wjm.2016.64010.

[9] A.A. Siddiqui, S. Ahmad, and M. Aqeel (2018), Influence of magnetic field on the merging flow of Powell-Eying fluids: an exact solution, Journal of Mechanica, 53(9), 2287-2298.

[10] M. Tadjfar, and F.T. Smith. (2004). Direct simulation and modeling of basic 3-dimensional bifurcating tube flow, Journal Fluid Mechanics. 519: $1-32$.

[11] A.R. Bestman. (1991). Global models for the biomechanics of green plants, part 1. International Journal of Energy Research. 19: 677-684.

[12] Erwin Kreyzig. Advanced Engineering Mathematics, John Wiley and Sons, Incorporation, New Delhi, India, 1983. 


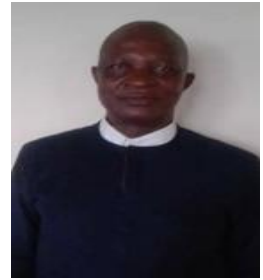

Professor Tamunoimi Michael Abbey was born on November 20, 1963. He had his primary education at St. Michael's State School, Bonny (1968 - 1974); Secondary education at Bonny National Grammar School, Bonny (1974 - 1979), where he obtained the West African School Certificate with Division one. Professor Abbey is an alumnus of three different Universities. He obtained a BSc (Applied Physics) with First class Honours from the University of Science and Technology, Port Harcourt in 1984; MSc (Semi-conductor electronics) from the University of Ibadan in 1987; Ph.D. (Applied Mathematics) with specialization in Magneto-hydrodynamics and plasma flows from the University of Port-Harcourt in 1995. He was appointed a Professor of Applied Mathematics and Theoretical Physics in 2008. He has held several challenging positions within and outside academics. $\mathrm{He}$ was the Director, Institute of Science Technology, University of Port Harcourt (2000-2004); a two times Head of Department of Physics (2004-2006 and 2014 - 2016); Vice President, Nigerian Universities Physics Writers Series; a visiting scholar to several academic centers including International Centre for Theoretical Physics, Italy. He is a member of several National and International organizations; an Editor of several local and international journals, and has over 152 articles in several national and international Journals. His published work (Steady MHD flow in a Bifurcating rectangular porous channel) is rated among the top best 500 articles on earth.

At present, he is the Rector of the Federal Polytechnic of Oil and Gas, Bonny Island, Nigeria. He is happily married to Barrister (Mrs.) Debonair Abbey and blessed with children.

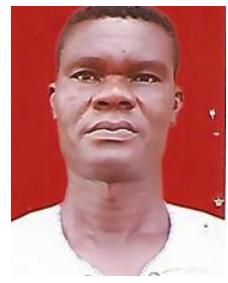

Dr. Wilson Ighoroje Ata Okuyade is a Nigerian of the Niger Delta extract, born on the $3^{\text {rd }}$ November 1960 at Ashaka. He had his elementary and higher school education at Iselegu Primary School, Iselegu (1970-1976), and St. Kizito's Grammar School, Utagba-Uno (1976-1981). He holds the degrees of Bachelors of Science Education (Mathematics) of the University of Port Harcourt (1992); Master of Science (Mathematics) of the Obafemi Awolowo University, Ile-Ife (2004); Master of Science (Applied Mathematics) of the University of Port Harcourt (2010) and Doctor of Philosophy (Applied Mathematics) of the University of Port Harcourt (2015). His researched interests are in Physiologic, Hydrologic, and Petroleum Reservoir flows; MHD; Heat, and Mass Transfer. He has published an upward of twenty-five (25) papers in reputable international Journals; reviewed about sixteen (16) manuscripts for two Journal houses: ScienceDomain International and International Knowledge Press. In collaboration with Prof. T. M. Abbey, he is the winner of the World Championship 2018 in Advances in Research (Bifurcating Rectangular Porous Channels), and a fellow of the International Agency for Standard and Ratings. He is rated as one of the World's 500 most influential experts in Mathematics on the planet Earth, and ascribed the title, 'Father of the Macular Hole' in Advances in Research.

He was the Dean of the School of Applied Sciences; now the Deputy Rector and Director of Academic Planning, Federal Polytechnic of Oil and Gas, Bonny Island, Nigeria. He is married to Mrs. Evelyn Okejepha Okuyade and they have four children: Oghenovo, Edesiri, Ohworode, and Eferhire.

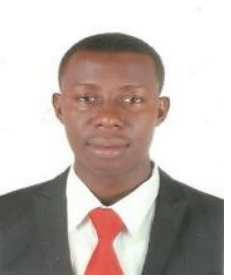

Mr. Minaibim Ellerton Abbey is a Nigerian of the Niger Delta extract, born on the $21^{\text {st }}$ September 1989 at Dema-Abbey Community. He had his primary and secondary school education at St. Michael Primary School, Bonny (1994-2000) and Community Secondary Oyigba, Ahoada East (2001-2007)

He holds a Bachelor of Science degree in Physics/Applied Geophysics at the University of Port Harcourt (2014); Master of Science degree in Applied Geophysics at Rivers State University, Nkpolu-Oroworukwo (ongoing); His researched interests are in Seismic, Resistivity, and Hydrology. He has published about Seven (7) papers in reputable international journals.

$\mathrm{He}$ is currently a lecturer in Physics, Instrumentation, and Process Control Technology in the School of Applied Sciences, Federal Polytechnic of Oil and Gas, Bonny Island, Nigeria. He is married to Mrs. Dabebara Minaibim Abbey.

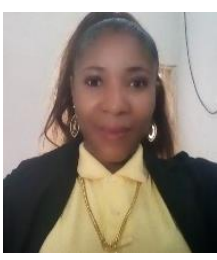

Mrs. Abbey Dabebara Minaibim is a native of Abonnema in Akuku-Toru Local Government Area of Rivers State, Nigeria. She had her elementary school education at Bishop Crowther Memorial School, Abonnema; secondary school education at Abonnema Girls' Secondary School, Abonnema. More so, she is an alumnus of the University of Port Harcourt and holds a Bachelor of Science degree in Biochemistry. She has published several papers. At present, she is a lecturer in Chemistry in the School of Applied Sciences, Federal Polytechnic of Oil and Gas, Bonny Island, Nigeria. She is married to Mr. Abbey Minaibim. 